\title{
Violencia de género: Una \\ aproximación desde el Análisis \\ de la Conducta
}

Rodríguez-Cira Maritza Ceciliaa ${ }^{1\left(^{*}\right)}$; Barrera-Torres Oswaldo ${ }^{1}$; Gajardo-Ayala David ${ }^{2}$

${ }^{1}$ Universidad Michoacana de San Nicolás de Hidalgo, Michoacán, México

${ }^{2}$ Universidad de las Américas, Región Metropolitana, Chile

\section{RESUMEN}

La violencia de género es considerada como una violación a los derechos humanos y un problema de salud pública por los efectos sobre la salud física, mental, sexual y reproductiva. La presente investigación es una revisión de literatura del año 2000 hasta el año 2021 sobre la violencia de género desde la perspectiva del Análisis de la Conducta. También, se describe el marco de referencia, su explicación sobre el fenómeno y aportaciones. Se considera la integración del Feminismo como actividad académica y política sobre la igualdad de género a favor de la garantía de los derechos para los hombres y las mujeres. Los resultados indican ausencia de artículos en Latino América desde la perspectiva mencionada, mientras que la literatura en idioma inglés se presentan cinco artículos. Se concluye que, son indispensables las aportaciones sociales de la ciencia culturo-conductual para explicar la interacción característica de la violencia contra la mujer (violencia doméstica o de pareja) y en general, para el estudio de la violencia de género.

Palabras clave: Violencia de género. Violencia doméstica. Feminismo. Análisis de la conducta. Cultura.

\section{Gender violence: Behavioral-Analytic Approach}

\section{ABSTRACT}

Gender violence is considered a violation of human rights and a public health problem due to its effects on physical, mental, sexual and reproductive health. The present research is a literature review from the year 2000 to the year 2021 about gender violence from the perspective of Behavior Analysis. It also describes the frame of reference, its explanation of the phenomenon and contributions. The integration of Feminism as an academic and political activity on gender equality in favor of the guarantee of rights for men and women is considered. The results indicate the absence of articles in Latin America from the mentioned perspective, while the English language literature presents five articles. It is concluded that, the social contributions to explain the characteristic interaction of violence against women (domestic or intimate partner violence) the inauguration of the culturo-behavioral science for the study of gender violence.

Keywords: Gender violence. Domestic violence. Feminism. Behavior analysis. Culture. 


\section{INTRODUCCIÓN}

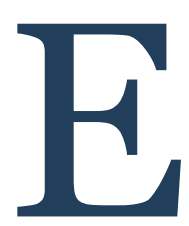

La violencia de género es definida como los actos dañinos (económicos, psicológicos, emocionales, físicos y sexuales) en contra de una persona o grupo por el motivo de su género principalmente, hace referencia a la estructura social en la que se conciben a las mujeres y niñas, incluyendo la población LGBTQI+, debido a que la violencia se ejerce con base a las normas de masculinidad y feminidad de cada cultura (ONU, 2021). Respecto a la violencia contra la mujer, se diferencia porque el acto es ejercido por la pareja o la familia, la OMS (2021) define la violencia contra la mujer como todo acto que resulte o pueda tener como resultado el daño físico, sexual y psicológico contra la mujer, inclusive las amenazas, la coerción o privación arbitraria de libertad en aspectos de la vida pública y privada. De tal manera que la violencia contra la mujer es un tema de relevancia social y conviene mostrar los datos que soporten su importancia. La Organización Mundial de la Salud (OMS) señaló que $35 \%$ de las mujeres ha experimentado alguna vez violencia física o sexual por parte de una pareja íntima o violencia sexual perpetrada por una persona distinta de su pareja (OMS, 2013). Años posteriores la ONU Mujeres (2020) reportó el incremento de hasta cinco veces de violencia contra las mujeres en el ámbito privado debido al aumento de

llamadas telefónicas, a causa de la restricción de movimiento, el aislamiento social y la inseguridad económica desencadenado por la pandemia de COVID-19 a nivel mundial.

La OMS (2021) reporta que una de cada tres mujeres (cerca de 736 millones) ha recibido violencia física y/o sexual a lo largo de su vida a nivel mundial, $27 \%$ de las mujeres entre 15 y 49 años admite haber sido víctimas de violencia física y sexual por parte de su pareja. La violencia contra la mujer (específicamente ejercida por la pareja y la violencia sexual) constituye un problema de salud pública debido a que los efec- tos de la violencia impactan sobre la salud física, mental, sexual y reproductiva. Asimismo, es considerado una violación a los derechos humanos. Sus repercusiones corresponden a la muerte, lesiones físicas, depresión, problemas de abuso de sustancias, infecciones de transmisión sexual (ITS), embarazos no deseados, abortos y el nacimiento de bebés con bajo peso (OMS, 2013).

En relación con América Latina, ONU Mujeres (2010) reporta en el Informe sobre Desarrollo Humano para América Latina que, prevalecen 10 de los 15 países a nivel mundial de desigualdad. La Comisión Económica para América Latina y el Caribe (2019) informa que, dos de cada tres feminicidios se producen en contextos de relaciones de pareja o ex pareja en América Latina. Los países con mayor incidencia de muerte por pareja o ex pareja son: República Dominicana, (1.5 casos por cada 100 mil mujeres), Uruguay (1.2 casos) y Paraguay (1.0). En el Caribe, se registró la mayor tasa de muertes en Trinidad y Tobago (1.7 por cada 100 mil mujeres) y Suriname (1.1). En cuanto a los feminicidios provocados por desconocidos, las tasas más altas por cada 100.000 mujeres se observan en el caso de Honduras (6,2), El Salvador $(3,3)$, República Dominicana $(2,7)$ y el Estado Plurinacional de Bolivia $(2,1)$. En México se reportan los datos provenientes de la Encuesta Nacional Sobre La Dinámica De Las Relaciones en los Hogares (ENDIREH). Señala que, la prevalencia total de violencia contra las mujeres fue de $66,1 \%$, los encuestados afirman haber sufrido al menos un incidente de violencia emocional, económica, física, sexual o discriminación a lo largo de su vida en al menos un ámbito y ejercida por cualquier agresor. Además, $41,3 \%$ sufrió violencia sexual, $29 \%$ violencia económica, patrimonial o discriminación en el trabajo, $34 \%$ violencia física y un $49 \%$ violencia emocional (INEGI, 2016). Otros datos relevantes son provenientes de la Encuesta Nacional de Seguridad Pública Urbana de México (ENSU), en los análisis del cuarto trimestre de 2020, demuestran que durante diciembre del 2020, 68,1\% de la población de 18 años y 
más, considera que vivir en su ciudad es inseguro dada la delincuencia; así también, el 33,3\% asegura que la delincuencia se mantendrá igual de mal para su país; el $14,3 \%$ de los encuestados señalan haber enfrentado alguna situación de acoso y/o violencia sexual en lugares públicos, el $13,1 \%$ señala pasar por intimidación sexual y el $17,6 \%$ de la población de mujeres encuestadas se les dijo piropos groseros $u$ ofensivos de tipo sexual o sobre su cuerpo que le molestaron u ofendieron (INEGI, 2021).

Referente a Chile, se reportaron los casos registrados por la Red Contra la Violencia contra la Mujer, donde CIPER cruza estos datos con la lista de feminicidios reconocidos por el del Servicio Nacional de la Mujer y Equidad de Género (SernamEG) y la información del Registro Unificado de Causas del Poder Judicial. Se analizó hasta el mes de febrero del 2021, señalando la existencia de 131 feminicidio, de los cuales solo el $14,5 \%$ ha sido resuelto judicialmente, el $51,1 \%$ sigue todavía en desarrollo, y el $6,8 \%$ se encuentra en estado de suspensión así también, el 29\% de los casos los imputados se suicidaron lo que provocó un sobre sentimiento de la causa antes de su dictamen, siendo solo en 3 casos acusados de cometer feminicidio (Segovia y Pérez, 2021). En lo que respecta a las denuncias registradas por violencia intrafamiliar hacia la mujer, Segovia y Pérez (2021) señalan que respecto a los análisis de llamadas al fono familia \#149 de Carabineros de Chile, la cantidad de víctimas ingresadas a las casas de acogida del SernamEG y las denuncias formales realizadas ante las policías, correspondientes entre enero y septiembre del año 2020, se han registrado un total de 24.806 llamadas pidiendo ayuda por violencia intrafamiliar. En el periodo Febrero-Septiembre de 2019 y 2020, respecto al Fono Familia posterior a septiembre 2020, se registraron 3.300 llamadas de auxilio y, en el fono ayuda para la Mujer se registraron 126, 645 Ilamadas de auxilio por violencia intrafamiliar contra la mujer, señalando una grave situación de vulneración a los derechos de la mujer en el país en la situación de pandemia vivida, esto refiere que, las condiciones de vida producidas por la pandemia han registrado un aumento en la violencia hacia la mujer en los hogares.

La violencia de género y la violencia contra la mujer son un problema latente y extendido durante décadas que ha requerido la respuesta por parte de los gobiernos, aunque eso ha sido por la influencia del feminismo (Walters, 2005). Mismo que es considerado, como toda teoría, pensamiento y práctica social, política y jurídica, que tiene por objeto hacer evidente y terminar con la situación de opresión que soportan las mujeres y lograr así una sociedad más justa que reconozca y garantice la igualdad plena y efectiva de todos los seres humanos (González, 2017). De acuerdo a Barriga (2020) el Feminismo se ha dividido por cuatro olas, las cuales se refieren a procesos y periodos determinados de tiempo en los que se distinguen por los reclamos y cuestionamientos sobre los derechos negados a las mujeres por razón de su condición biológica. Se evidenció la "superioridad" masculina impuesta en diversas áreas de la vida pública y privada (política, economía, educación, entre otras). La primera ola (S. XVIII al S. $X I X)$, su representante principal François Poulain de la Barre aportó tres obras importantes: 1) De la igualdad de los sexos, 2) La educación de las damas para la conducta del espíritu en las ciencias y las costumbres, y 3) La excelencia de los hombres contra la igualdad de los sexos. Además, Olympe de Gouges realizó La declaración de los Derechos de la Mujer y la Ciudadana y Mary Wollstencraft escribió el libro: Vindicación de los derechos de la mujer y los Cuadernos de Queja los cuales se escribieron en 1789 por mujeres de diversas clases sociales. Dichas obras dispusieron la manifestación de las mujeres en protesta contra la jerarquía a la que eran sometidas socialmente a través de la historia. La segunda ola comenzó en la segunda mitad del S. XIX hasta el primer tercio del S. XX. Esta etapa es considera como: sufragista debido a que se centró en la participación de las mujeres en el ámbito político. Además de acceder a la educación de la misma manera que 
los hombres. De manera consiguiente, la tercera ola inició en la segunda mitad del S. XX y terminó en el inició del S. XXI. Este periodo es encabezado por Simone de Beauvoir con su obra: El segundo sexo. Simultáneamente Betty Friedan escribió las obras: La mística de la feminidad y La segunda fase, sus obras destacadas para el feminismo. El objetivo de la tercera ora consistió en la manifestación de los derechos sexuales y reproductivos de las mujeres. La última ola es vigente, surgió en el S.XXI. Sus objetivos son la erradicación de la violencia contra las mujeres en todas sus modalidades, los eventos hechos históricos que demuestran su objetivo, los movimientos iniciaron en 2011, el día 13 de febrero del mismo año, en el que cantidad masiva de mujeres en Italia se movilizaron en favor de su dignidad y protestaron por ser consideradas objetos sexuales, con esta frase en representación: "si no ahora cuando" (se non quando). Hechos posteriores el 3 de junio del 2015, hubo una manifestación en Argentina frente a la violencia machista y el feminicidio con la consigna: "ni una menos", nuevamente, el 3 de junio del 2016 se manifestó la multitud con la frase: "vivas nos queremos" y con "basta de violencia machista y complicidad estatal" el 3 de junio del 2017. Por último, cabe señalar que, el 8 de marzo del mismo año, mujeres de 57 países participaron en el primer paro a nivel mundial de mujeres.

La presente investigación muestra la aproximación del análisis de la conducta como otra propuesta para comprender y explicar la violencia de género, la violencia contra la mujer y su tipología (violencia física, emocional, sexual, económica y psicológica) mediante una revisión de artículos publicados sobre el tema.

\section{MARCO TEÓRICO}

La violencia de género ha sido estudiada por una variedad de ciencias, sin embargo, dentro de la Psicología, el Análisis de la Conducta es otra propuesta alternativa para su comprensión. A conti- nuación, se describen sus características y su relación con la violencia de género.

El Análisis de la Conducta $(\mathrm{AC})$ es un enfoque de las ciencias naturales que considera como su objeto de estudio la conducta, es decir, el estudio de la actividad de los organismos en relación con su medio ambiente (Skinner, 1974). La ciencia del comportamiento se constituye de tres disciplinas (Cooper et al., 2007): 1) El Análisis Experimental del Comportamiento, la cual es responsable de la investigación básica del comportamiento mediante diseños de investigación experimental; 2) El Análisis Conceptual del Comportamiento que analiza, critica y provee teorías y filosofía de acuerdo a los conceptos fundamentales del Análisis de la Conducta en relación a los datos obtenidos por las investigaciones; y 3) El análisis conductual aplicado, su objetivo consiste en realizar investigación aplicada con la intensión de solucionar problemas socialmente relevantes. Cada disciplina está relacionada y sus aportaciones dependen de la actividad de cada campo.

La perspectiva del análisis del comportamiento se considera un enfoque funcional debido a que estudia la actividad del organismo, en vez de considerarlo como objetos o entidades internas de las personas en términos de sustantivos, dentro de Psicología, se refiere a las expresiones lingüísticas en las que se considera el comportamiento como objeto (Freixa, 2003). Se retoma aquí, la perspectiva del Análisis de la Conducta y su relación con la violencia de género debido a que se considera como actividad, como acciones relacionadas a situaciones o contextos, en vez de atribuirles características o rasgos propios o internos como la causa del comportamiento. El problema principal respecto al tema tiene que ver con la concepción del abusador o agresor, es explicado conforme a su personalidad, manejo de emociones, entre otras, el dilema supone las siguientes preguntas: ¿Cómo y por qué actúa de esa manera? La explicación de que el abusador es machista o misógino es adecuada. Sin embargo, 
carece de elementos explicativos que puedan ser accesibles para su estudio científico porque sólo enuncian una causa aparente, sólo se nombra el problema. La conclusión que conlleva el argumento critica la concepción simplificadora y sobre individualizada del comportamiento del abusador a través de explicaciones alusivas a elementos dentro de las personas como categorías causales. Enunciar que un hombre es machista, sólo indica de qué manera se comporta conforme a la convención social. El estudio científico de la violencia de género implica, entonces, más que describir y nombrar fenómenos. La concepción del Análisis de la Conducta sobre la violencia de género es la actividad individual en relación con el ambiente social y cultural.

La violencia de género desde el punto de vista del Análisis de la Conducta es una práctica cultural e individual mantenida por las instituciones de acuerdo a la denominación de género (hombre y mujer). Su aportación, por un lado, es la explicación de la interacción de pareja usualmente denominado como abuso doméstico, violencia doméstica o violencia de pareja y por el otro, la explicación del comportamiento cultural relacionado a los aspectos sociales (instituciones, leyes, costumbres, etc.) y las prácticas de sus miembros como el sexismo (discriminación a partir de prejuicios determinados por criterios sociales sobre el género).

\section{Análisis de la conducta social y cultural de la violen- cia de género}

Skinner (1953) postula que "El comportamiento social puede definirse como el comportamiento de dos o más personas entre sí o en conjunto con respecto a un entorno común" (p. 297). El concepto de conducta social (social behavior) es el comportamiento individual en relación con otros individuos y grupos mediante el reforzamiento social (social reinforcement) y su relación con la conducta verbal (verbal behavior). La conducta social se determina a partir de la observación del comportamiento de cada individuo en conjunto y las relaciones funcionales que establecen en un episodio social (social episode), de manera que, se describen los sistemas de acción interrelacionados y sus efectos (funciones estímulo) multilaterales dentro de un evento o situación particular. La violencia contra la mujer supone un episodio social entre dos miembros (pareja) y en ciertos casos, más miembros que conforman una familia. Cada miembro responde de manera dependiente ante las respuestas y las situaciones públicas y sobre todo privadas en las que se involucran a causa de la mediación de sus actos por el uso de palabras y objetos.

Skinner (1957) propone el concepto de conducta verbal para explicar los efectos de conducta escrita, hablada o señalada (referida a los signos) en la comunicación con otros individuos por la mediación de sus acciones para alterar el ambiente dentro del episodio social. Dicho de otra manera, la conducta social depende la relación de otros individuos designados como estímulos sociales (social stimulus) y su relación para la disposición de reforzadores, principalmente aquellos denominados reforzadores secundarios o condicionados (conditional reinforcement) a través de la comunicación de palabras habladas, escritas o señaladas de otros individuos como hablantes, oyentes y observadores.

De la misma manera, el comportamiento se clasifica por los efectos derivados de los tipos contingencias: a) Conducta moldeada por contingencias (contingency-shaped behavior), el comportamiento es alterado por el contacto directo con las contingencias de reforzamiento de las situaciones $\mathrm{y}, \mathrm{b})$ Conducta gobernada por reglas (rule-governed behavior), en el que el comportamiento es alterado por el contacto indirecto de contingencias de reforzamiento derivadas de la conducta verbal (Vaughan, 1989). Concretamente, la violencia de género desde el aspecto de la conducta social, supone analizar el comportamiento del hombre y los efectos que causa sobre la mujer. Por ejemplo, las restricciones, las amenazas, 
el abuso físico y las manifestaciones verbales en forma de críticas y ofensas. Simultáneamente, el comportamiento de la mujer ante la situación de abuso supone efectos sobre el hombre como por ejemplo cumplir con las demandas para evitar el abuso, idear un plan para escapar o abandonar y confrontar al abusador.

La conducta social y la conducta verbal son interdependientes para describir el control personal de dos o más personas, aun así, el comportamiento obedece una organización de nivel superior en el que se analizan los sistemas sociales y grupos designados como cultura. A continuación, se reseñan los conceptos para explicar la violencia de género a nivel cultural. Skinner (1981) propuso el término de selección por consecuencias (selection by consequences) para describir tres niveles de análisis del comportamiento, 1) nivel biológico, se concibe el aspecto genético y el proceso de selección natural; 2) nivel conductual, relacionado con la ontogenia y el condicionamiento operante como proceso de selección de comportamientos individuales; y 3 ) nivel cultural alusivo a las prácticas en sociedad y su mantenimiento durante generaciones (Glenn, 1989).

Skinner (1984) propone el concepto de cultura como conjunto de comportamientos mantenidos por las contingencias sociales de reforzamiento dispuestas por un grupo particular, específicamente la comunidad verbal referida a la conducta de los grupos que regula la conducta verbal en relación con las prácticas y costumbres de un oyente y un hablante. Glenn (1991) explica que las prácticas culturales pueden incrementar y mantenerse o en su defecto, disminuir a partir de los efectos o resultados de un conjunto de individuos bajo los principios del comportamiento operante, la interrelación de individuos establece el tipo de estimulación y reforzamiento de las acciones de otros. Dicho concepto se denomina: contingencias conductuales entrelazadas (interlocking beha- vioral contigencies) y sus efectos denominados como producto agregado (aggregate product). Las contingencias conductuales entrelazadas y el producto agregado conforman la unidad de análisis designada como metacontigencia (metacontigency). Bajo esta premisa, la violencia de género es la práctica general de las personas dentro de las instituciones en la que se distinguen de la relación de pareja porque sus efectos sobre la mujer son vistos por la incidencia de denuncias de violación y de acoso sexual. También se distingue el comportamiento en pareja del comportamiento cultural porque las practicas afectan a las mujeres de manera sistemática, estructural provenientes de un orden institucional. Por ejemplo, la desigualdad de salarios, la imposición profesional conforme a la convencionalidad del género, los derechos laborales, la cantidad de mujeres involucradas en actividades políticas, las manifestaciones realizadas por mujeres en las exigen igualdad y el cumplimiento de sus derechos, entre otros.

El presente trabajo pretende demostrar la posibilidad de estudiar la violencia de género desde la perspectiva analítico-conductual mediante la revisión e identificación de investigaciones desarrollados y publicados. La investigación presenta tres objetivos en la revisión de artículos: 1) Reportar la cantidad general de publicaciones en psicología en relación con la violencia de género, 2) Reportar la cantidad de publicaciones en análisis de la conducta relacionados con la violencia de género y 3) Describir los aportes teóricos y metodológicos de la violencia de género desde la perspectiva del análisis de la conducta.

Se ha demostrado el valor de estudiar la violencia de género y la perspectiva del análisis de la conducta es otra posibilidad. Además, se han reportado una serie de aportaciones derivadas del análisis de la conducta relacionadas con problemas globales como el racismo (Matsuda et al. 2020, Saini y Vance, 2020; Barrera-Torres et al. 2021) y el cambio climático (Todorov, 2010; Luke y Alavosius, 2012). 


\section{METODOLOGÍA}

La presente revisión de artículos se realizó de manera manual en tres bases de datos: a) Redalyc, b) Scielo y c) Scholar Google durante el mes de mayo del presente año considerando un margen de rango a partir del año 2000 hasta el 2021. Los criterios de inclusión consistieron en: 1) Artículos teóricos (conceptuales) y empíricos, 2) Los artículos son explícitamente en el tema del análisis de la conducta y 3 ) Los artículos están relacionados con los temas de violencia de género y violencia doméstica o violencia de pareja. Por último, se consideraron artículos en los idiomas español e inglés.

El proceso de búsqueda empleó las palabras clave y operadores de búsqueda de la siguiente manera: "violencia de género";" psicología"; análisis de la conducta". Primero, se realizó una búsqueda general de artículos de todas las disciplinas disponibles. De manera consiguiente, se delimitaron los artículos de interés en el área de Psicología y por último, se seleccionaron los artículos sobre Análisis de la Conducta. Por lo que respecta a los criterios de exclusión: 1) se descartaron los artículos derivados de otras disciplinas, a pesar de investigar el tema de violencia de género, y 2) trabajos de grado (tesis y tesinas).

Los resultados serán presentados en el siguiente orden: a) Número de publicaciones totales dentro del margen señalado; b) Número de publicaciones totales en el área de psicología; c) Número de publicaciones totales en análisis de la conducta; d) Número de publicaciones por cada año en el área de psicología.

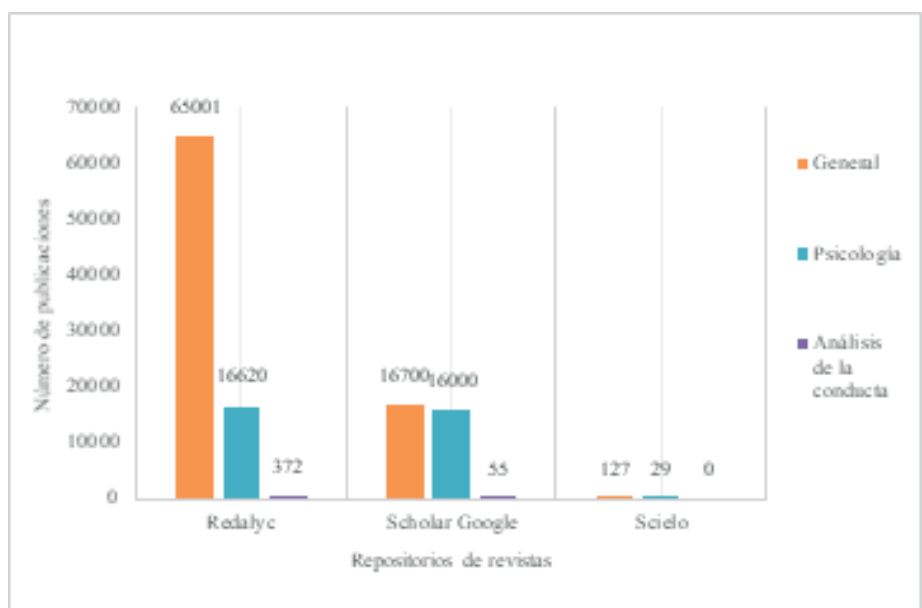

Figura 1. Número total de publicaciones del año 2000 a 2021 sobre violencia de género
vLos resultados demuestran que el número total de publicaciones durante el año 2000 hasta el 2021. Redalyc muestra un total de 650001 artículos publicados, Scholar Google un total de 16700 y Scielo 127 artículos publicados sobre violencia de género desde varias disciplinas. Por lo que refiere a la cantidad de artículos publicados en el área de psicología Redalyc muestra 16620, Scholar Google 16000 y Scielo 29 artículos sobre violencia género. En resumen, la cantidad de publicaciones en general es mayor a la cantidad de publicaciones en el área de psicología. Por último, la cantidad de publicaciones en Análisis de la Conducta y violencia de género muestran que Redalyc publicó 372 artículos sobre análisis de la conducta, Scholar Google 55 artículos y Scielo 0 artículos. A continuación, se reportan la cantidad de artículos publicados por cada año en el área de psicología.

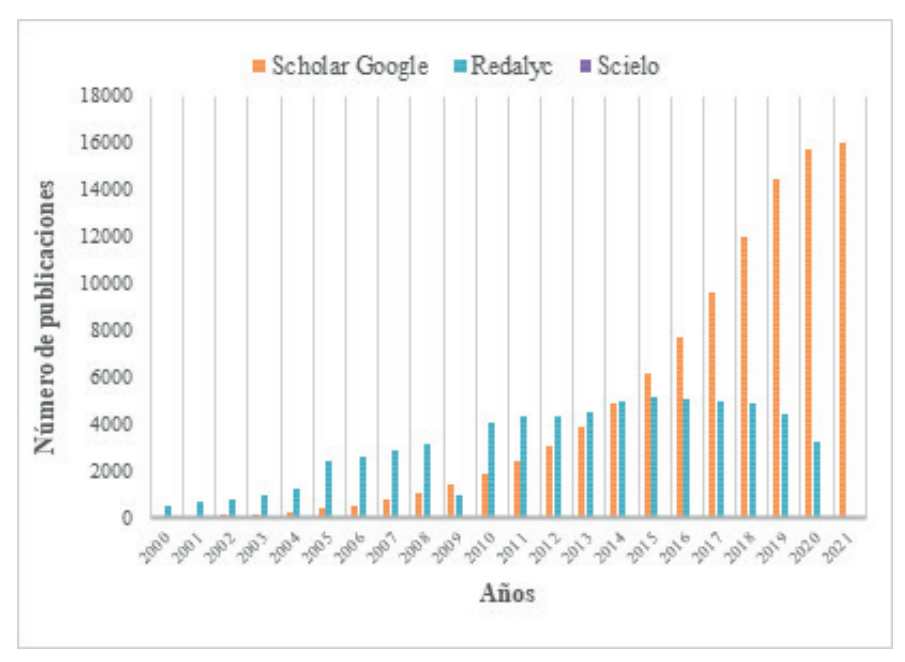

Figura 2. Número de publicaciones por año de cada repositorio de búsqueda

Los resultados de búsqueda sobre la cantidad de artículos publicados por año en el área de Psicología, muestran una cantidad menor a 2000 artículos del año 2000 a 2004. Desde el año 2005 hasta el actual 2021, se ha incrementado la cantidad de publicaciones en los tres repositorios de búsqueda. Redalyc presenta una cantidad de 6000 artículos publicados y es menor con respecto a Scholar Google que ha incrementado la cifra mencionada en el año 2015 hasta las 16000 publicaciones en el presente año. Por otro lado, Scielo ha publicado menos de 100 artículos. 
Los artículos encontrados y seleccionados en la búsqueda son cinco en idioma inglés. En seguida, se muestra el resumen de los trabajos identificados en tres secciones: a) Conceptualización del término género desde el Análisis de la Conducta; b) Comportamiento a nivel individual en la violencia doméstica o de pareja; y c) Comportamiento a nivel cultural.

\section{RESULTADOS Y/O DISCUSIÓN}

\section{Fuentes imperceptibles de control del comporta- miento: El caso de las prácticas de género}

Ruiz (2003) plantea que "El género como una construcción social de conocimiento moldeado y mantenido por contingencias sociales y patrones de reforzamiento dentro de la comunidad verbal" (p.12). El artículo Inconspicous sources of behavioral control: The case of gendered practices señala que el Análisis de la Conducta se ha desinteresado por las cuestiones de género. La postura del Análisis de la Conducta considera el género a partir de la conducta de los individuos, en vez de considerarlas como una característica propia, se refieren a las prácticas culturales que han sido asociadas como categoría biológica. La autora distingue tres consideraciones teóricas: 1) El dualismo distinguido entre el individualismo y el yo (the self) como un locus o agente de acción, 2) dualismo referido en el trabajo como la designación basada en un criterio de tipo sexual basada en la distinción en categorías biológicas en particular, se refiere a la caracterización como mujer u hombre en virtud de su sexo, y 3) el dualismo constituido en la comprensión de los aspectos femeninos y masculinos. La presente aportación conceptual se deriva de considerar el género (categorización de hombre o mujer) como un estímulo discriminativo en relación con la conducta de otros individuos por medio de la conducta verbal y la imitación de los observadores dentro de las instituciones, considerando así las diferencias entre contingencias dispuestas en favor de los hombres y el control sobre las mujeres.

Entendiendo las decisiones de permanecer/abandonar en las relaciones violentas: Un enfoque analítico conductual
Bell y Naugle (2005) realizaron su investigación con la intención de extender la propuesta de (Myers, 1995) sobre la conceptualización de violencia de pareja. Los propósitos del artículo corresponden a describir tres teorías principales que conceptualizan el fenómeno: 1) la teoría de la indefensión aprendida (learned helplessness), 2) la teoría de la trampa psicológica (psychological entrapment), y 3) la teoría de la inversión (investment theory). Las teorías están basadas en el supuesto de que las mujeres dentro de la relación de abuso realizan la toma de decisión de manera racional. Tales términos se refieren a un proceso decisión de una víctima basado en la evaluación de datos disponibles usando reglas de decisión predecibles.

La teoría de la indefensión aprendida propuesta por Martin Seligman establece que la aparición consecutiva y repetida de estímulos aversivos deriva a la expectativa de consecuencias con independencia de las respuestas, Es decir, se refiere a que la mujer presentará expectativas de recibir castigos sin importar los intentos y las posibles respuestas para evitar y escapar del abuso doméstico. Walker (1979) retoma el modelo teórico de la indefensión aprendida para explicar la dinámica de las relaciones de pareja y el abuso doméstico a partir de la consignación social de roles de género imperativas, su efecto sobre la mujer concierne al hacer creer que es inevitable escapar de la relación. Por lo que respecta a la teoría de la trampa psicológica, propone que el nivel de compromiso dentro de una relación aumentará a medida que aumente la cantidad de inversiones en esa relación en términos de tiempo, energía, dinero, entre otros. Retomando dicho argumento, la mujer en contexto de violencia domestica permanece dentro de la relación para justificar las acciones previas de su inversión y conseguir una relación adecuada, considerando el supuesto de que el comportamiento de la relación se desempeñó con propósitos y motivos explícitos dirigidos hacia una recompensa, meta o expectativa particular.

Finalmente, la teoría de la inversión sustentado en la teoría del intercambio (exchance theory) de John 
Thibaut y Harold Kelley describe la violencia doméstica en términos de un análisis costo-beneficio para la toma de decisiones por parte de la mujer, basados en la comparación de costos y recompensas que implica la relación de pareja ante un incidente de abuso sobre la estimación de los beneficios y costos de relaciones alternativas que implica dejar o abandonar la relación. Según el modelo de la inversión es determinado por nivel de compromiso que tiene la mujer depende de tres factores: grado de satisfacción con la relación, calidad de las alternativas disponibles e inversión realizada. Intervienen dos procesos, el primero está relacionado con la estimación de la relación actual en términos de satisfacción (beneficios y recompensas de la relación: dinero, hogar, alimentos, etc.) y el segundo proceso conlleva comparar el número total de costos asociados a las situaciones de maltrato (frecuencia de conflictos, severidad del abuso, inestabilidad, etc.).

De ahí que la teoría de la inversión consiste, básicamente, en el principio de comparar los niveles de satisfacción personal involucrados en la relación y los inconvenientes que implica. De acuerdo al modelo, las mujeres emplean cuatro estrategias de afrontamiento para sobrellevar el abuso doméstico. Primero, denominado como respuesta de autocastigo (self-punishing response) en la que la mujer cree que es responsable de estar sometida en la relación y carecer de opciones alternativas. En otras palabras, el costo de abandonar al abusador supone la evaluación en términos de perdida, de manera que la mujer termina culpándose asimismo por los incidentes de abuso físico, emocional, económico y psicológico. Segundo, la estrategia de afrontamiento denominada como respuesta agresiva (aggressive response), en la que la mujer toma represalias contra su pareja y los hijos a causa de altas retribuciones por parte de la pareja, pero bajas retribuciones de relaciones alternativas. Tercero, la respuesta de desvinculación como estrategia de afrontamiento (disengagement response), consiste en la descripción de la toma de decisiones en la que la pareja presenta bajas retribuciones y las relaciones alternativas conllevan retribuciones altas, la estrategia de afrontamiento establece que dicha toma de decisión implica el abandono y la retirada de la relación de manera más probable. Por último, la respuesta reticente de desvinculación a media vida (reluctant mid-Ife disengagement response) indica que las retribuciones son altas en ambos aspectos dentro de la relación y fuera de ella, por lo que, la toma de decisión es la retirada gradual debido a los riesgos que están en la relación. Los autores proponen la conceptualización del Análisis de la Conducta para explicar el comportamiento de toma de decisiones en términos de triple relación de contingencias (antecedentes, conductas y consecuencias) y principios básicos del comportamiento: reforzamiento positivo y negativo (efecto de incrementar la conducta por la presencia o removimiento de objetos y situaciones), castigo positivo y negativo (decremento del comportamiento por la presencia de estímulos aversivos y el removimiento de estímulos reforzadores) y las operaciones motivacionales. Conviene subrayar que las investigaciones en los años posteriores comparten la similitud de considerar dichos conceptos básicos en condicionamiento operante: 1) los déficits en los repertorios conductuales del abusador para atender problemas de la vida cotidiana, 2) los estímulos discriminativos señalados a través de amenazas verbales (por ejemplo, si me dejas te mataré) y 3 ) las operaciones motivacionales que afectan la efectividad de la clase operante de abandonar la relación.

Considerando que objetivo del artículo es extender e incorporar otros modelos contemporáneos basados en el Análisis de la Conducta. Los autores mencionan la inclusión de las teorías en economía conductual para explicar la conducta de decidir y seleccionar reforzadores dependiendo de factores como la tasa de reforzamiento, probabilidad de reforzamiento y a los criterios de esfuerzo de estímulos reforzadores (pauta o condición que se requiere para la obtención de un reforzador). Para concluir, se enfatiza la necesidad de realizar investigaciones básicas y aplicadas en torno a la conceptualización de principios de condicionamiento operante y los modelos de economía conductual para explicar la relación de abuso doméstico.

Aplicación del aprendizaje operante a la decisión de 
quedarse o irse en caso de violencia doméstica

Miller, Lund y Weatherly (2012) en su investigación Applying operant learning to the stay-leave decision in domestic violence, proponen un análisis conceptual en términos de condicionamiento operante sustentado en datos experimentales de análisis de la conducta y otras investigaciones sobre la violencia doméstica. La presente investigación es una propuesta alternativa ante las investigaciones sobre factores (económicos, psicológicos, ambientales y de apoyo social) que determinan la toma de decisión de mujeres respecto a quedarse o alejarse de la relación íntima en contextos de violencia doméstica.

La explicación de la violencia doméstica varía de manera circunstancial a partir de las historias de reforzamiento de cada miembro de la pareja o la familia respecto a sus repertorios conductuales para establecer comunicación y solucionar problemas. De acuerdo a los autores, la explicación del abuso doméstica en la perspectiva del Análisis de la Conducta depende de los siguientes factores individuales-situacionales en la relación de abuso a partir de las contingencias dispuestas por el abusador: 1) Las contingencias de reforzamiento intermitente (reforzadores positivos y negativos), 2) la disminución de la efectividad del castigo y 3 ) las contingencias de castigo ante los intentos de escapar de la relación explican cómo es que las mujeres se mantienen y "permanecen" en la relación de abuso doméstico.

La violencia doméstica es conceptualizada principalmente por la autora Lenore E. Walker en 1974, su modelo propone un ciclo compuesto por tres fases: 1) Acumulación de tensión (tension building), 2) Comportamiento abusivo o incidente (abusive behavior) y 3) Reconciliación (reconciliatory behavior and promises to change) con las que explica el comportamiento de abuso doméstico (Walker, 1979). Primer punto, los parámetros del reforzamiento intermitente o parcial (intermittent reinforcement) y el reforzamiento continuo (continuos reinforcement) son los que determinan la decisión de quedarse debido a la impredictibilidad del comportamiento del abusador a causa de que el abusador se comporta conforme a la situación de abuso. Es decir, el abusador actúa de manera "amable", "respetuosa" y "afectiva" una vez ocurrido el incidente de abuso físico (principalmente) en las situaciones de "calma". Durante la fase de reconciliación el abusador realiza promesas o juramentos de cambio, verbalizaciones de tipo afectiva en el que dice amar o querer a la mujer, actúa conforme a las expectativas que conforman el problema (usualmente "cambia" la persona en su manera de hablar para solicitar o expresar sus inconformidades, se compromete con las tareas de casa, muestra señas de afecto, entre otras). Concretamente, el afecto es un reforzador social para el control de la mujer debido a su historial previo en el que se estableció la relación de pareja, dichos principios explican el mantenimiento de la mujer en la relación. Además, el afecto puede cumplir la función estímulo de una operación motivacional que altera los eventos de reforzamiento negativo de la conducta de alejarse o irse. Los actos de violencia doméstica se presentan de manera gradual, a pesar de que la relación de pareja en un principio era caracterizada por tratos "cálidos", "caballorosos" y "cariñosos". Por lo que refiere el principio del reforzamiento negativo, está relacionado con los comportamientos de evitación y escape de eventos de situaciones aversivas (estímulos aversivos condicionados) en el cual se incrementa la probabilidad de tales comportamientos. El contexto de la violencia doméstica se anticipa o previene la situación de abuso a manera de demandas y peticiones sobre el comportamiento de la mujer. Particularmente, la mujer hará lo que espera o solicita el hombre a condición de evitar por completo o reducir la severidad del abuso (intensidad). Por lo tanto, el comportamiento del abusador es anticipado para prevenir la "penalización", se evitan los tratos inadecuados de las situaciones en las que se involucran las demandas o peticiones. De ahí, el control se extiende en la mujer en actos como el mantener distancia o perder contacto con personas a petición del abusador, mentiras sobre cómo se causaron ciertas lesiones o afirmar que se encuentra satisfecha y contenta con su relación.

Segundo punto, los eventos aversivos y las contingencias de castigo determinan su efecto en el decre- 
mento de la probabilidad del comportamiento. Usualmente, el término castigo es empleado para referirse a reprimendas o sanciones, desde el punto de vista presente, los castigos sólo se definen a partir de sus efectos sobre el comportamiento. Los efectos del castigo son: a) La intensidad alta reduce inmediatamente el comportamiento, b) La intensidad gradual e intermitente de castigos positivos y negativos incrementan (paradójicamente) la frecuencia del comportamiento y presentan habituación de respuestas emocionales y c) El castigo y sus procedimientos (tiempo fuera, sobrecorreción, práctica positiva, coste de respuesta, etc.) están relacionados con conductas reforzadas (se reducen o se pausan únicamente). El abusador emplea el "castigo" en el sentido moral porque trata de "reducir" ciertos comportamientos de la mujer que son considerados inadecuados o incorrectos conforme a concepciones sociales de lo que debe hacer o debe ser una mujer. La violencia doméstica incrementa su intensidad de manera gradual y se establece un grado de control en el que la mujer es imposibilitada a dejar la relación por los distintos aspectos que implica, por una parte, la relación y las repercusiones directas sobre la mujer en el caso de tomar una de decisión de retirada.

Tercer punto, el coste de respuesta es un procedimiento fundamentado en la contingencia de castigo, el problema de dejar la relación está relacionado con el hecho de que una conducta alternativa (irse o alejarse del abusador) es decrementada a partir de los criterios "altos" o "excesivos" de la situación. El coste de respuesta consiste en remover estímulos reforzadores condicionados (objetos u eventos que por la experiencia de la persona son condicionados) y sus efectos son la reducción del comportamiento. Conforme a lo explicado, el coste de respuesta implica para la mujer confrontar una serie de situaciones como denunciar o realizar un reporte en contra de su abusador, buscar refugio o un lugar seguro, contactar amigos y familiares para solicitar ayuda, además de explicar la situación de abandono, realizar un plan para escapar, realizar los procedimientos legales correspondientes a la custodia de los hijos, el divorcio, orden de alejamiento (restricción) e inclusive estar bajo la condición de amenaza de muerte (entre otros). Dichos efectos también pueden ser observadores en efectos indirectos mediante el daño de un tercero por parte del abusador como el secuestro o maltrato de los hijos y el maltratado hacia mascotas (consideradas como miembros de la familia).

\section{Etiquetado de los actos de violencia sexual: ¿Qué papel desempeñan las características de la agre- sión, las actitudes y las experiencias vitales?}

Sasson y Paul (2014) en su investigación Labeling acts of sexaul violence: What roles do assault characteristics, attitudes, and life experiences play?, se interesan por la influencia de la categorización de la violación sexual a través de la percepción de mujeres en el reconocimiento y descripción de los hechos de un acto de violación sexual. De acuerdo a la investigación, la concepción de violación sexual implica la agresión o actos de violencia por parte de un hombre en el que usa la fuerza física o un arma para controlar la resistencia de una mujer desconocida. A partir de dicha consideración autores señalan inconsistencias y la describen como características estereotipadas de la violación (stereotypical rape characterictics), los autores demuestran que, en general, las personas conciben la violación sexual con las características descritas previamente. Sin embargo, datos obtenidos por víctimas de violación sexual reportan una relación interpersonal con el abusador y los hechos descritos carecen de similitud con las características generales atribuidas. (La violación sexual es ejecutada por un desconocido a través de medios coercitivos como la fuerza física y el uso de armas en un evento súbito). La relevancia sobre el reconocimiento para describir el acto de violación puede presentar efectos positivos, por una parte, para las víctimas no reconocidas y expongan el incidente a sus familiares y amigos, la atención de aspectos psicológicos (depresión, ansiedad, estrés post-traumático, entre otras) y la prevención de víctimas mediante la disposición de apoyo y servicio social. De modo que, la descripción y reconocimiento de uno mismo como víctima de abuso sexual es una medición que permite la estimación de la prevalencia de la violación y la violencia sexual. 
El objetivo de la investigación consistió en analizar los factores que influencia la etiquetación de un acto de violación sexual para incrementar los conocimientos posibles en beneficio de las víctimas. Su diseño de investigación consistió en la aplicación de una encuesta en línea a 401 participantes con una duración de 20 a 30 minutos y una compensación monetaria, además de emplear un consentimiento informado para proceder. La encuesta consistió en 16 viñetas intercambiadas dentro de un relato de hechos de violación sexual, se manipularon las viñetas respecto a la identidad del perpetrador (extraño o conocido), uso de la fuerza (sí o no) y uso de la resistencia (verbal, física, ambas o ninguna) los cuales se presentaron de manera individual con las opciones de respuesta dicotómica (sí o no). En total, se conformaron un aproximado de 125 palabras con la intensión de describir un acto de violación con la finalidad de medir la percepción de los participantes a partir de las manipulaciones descritas, se presenta a continuación las viñetas empleadas:

"Alrededor de las 8:30 p.m. del día lunes, Melissa se retiraba de su oficina para ir a su casa por la noche. Mientras caminaba hacia su automóvil, se percató que un hombre/su colega, Michael se acercaba detrás de ella. Ella nunca ha visto al hombre anteriormente/ Ella ha trabajado cerca de Michael por al menos un par de años y ha disfrutado salir con él y sus colegas del trabajo después de la oficina. El hombre/ Michael dijo hola al acercarse a su auto (automóvil de Melissa). Ella responde y mientras lo hace, él la toma alrededor de la cintura y busca el botón de sus pantalones. Mellisa grita; ino, detente! ¡Déjame ir!/ Lucho para alejarse de él/ grita; iNo, detente! iDéjame ir! Lucho para alejarse de él/Se congelo/ pero/y él la amenaza de muerte si ella no se detiene. El hombre/ Michael la obligó a tirarse al suelo, le inmovilizó los brazos bajo la espalda y le bajó de un tirón los pantalones y la ropa interior. A continuación, la penetró y mantuvo relaciones sexuales con ella. Cuando el hombre/Michael terminó, huyó dejando a Melissa sola junto a su coche".

Las cursivas empleadas señalan las viñetas manipuladas por los investigadores, el relato se compartió con un orden aleatorio de las viñetas. Para analizar los datos, emplearon un estadístico de chi-cuadrada para relacionar la posible asociación de dos variables (características del asalto, variables actitudinales y diferencias individuales) y una regresión logística para predecir la categorización de violación (predicción de categorización de violación sexual y asignación de responsabilidad). Las conclusiones demuestran que el estudio incorpora datos adicionales sobre los factores que influyen en cómo se percibe la violencia sexual y su categorización por otros, la descripción del incidente es un factor relevante para considerarlo violación sexual. Por otra parte, la conceptualización de patrones estereotípicos de violación es consistentes con la literatura, aunque a que considerar que las nociones sobre violación sexual cambian con el paso del tiempo, los investigadores señalan que el reconocimiento de violación sexual por el público general puede modificar el comportamiento del agresor para evitar su identificación, muestra de ello es emplear el abuso emocional y psicológico en vez del abuso físico. Otra consideración reside en la disposición limitada de variables en la encuesta. Por último, las implicaciones del análisis de la conducta se relacionan directamente con los sistemas judiciales y legales, ello implica la propuesta de intervenciones a nivel institucional para la atención a víctimas de violación sexual.

\section{El futuro es femenino ( $y$ el análisis del comporta- miento): Un relato conductual del sexismo y cómo el análisis de la conducta es simultáneamente parte del problema y de la solución}

Baires y Koch (2020) realizaron una investigación sobre la intervención de las mujeres dentro del Análisis de la Conducta y el sexismo como un problema que afecta la actividad de la investigación dentro de la disciplina del comportamiento y el sexismo como problema social y cultural a nivel global. Las autoras incorporan el punto de vista del feminismo de la cuarta ola descrito como el movimiento que trata eliminar el sexismo garantizando los derechos sociales, económicos y políticos para todos los géneros. Su aproximación conductual refiere a la ciencia del comportamiento de la cultura en términos de meta- 
contigencias como unidad de análisis, las contingencias conductuales entrelazadas como la relación de la conducta de varios individuos y su efecto denominado producto agregado, el cual determina si las prácticas culturales se presentan con probabilidad en el futuro o en efecto su decremento. De acuerdo a su investigación, retoman las ideas de (Ruiz, 2003) para sustentar la relación de género y el estudio de la actividad individual de los organismos. Con respecto al sexismo, el Análisis de la Conducta considera que son prejuicios y estereotipos que implican prácticas culturales emitidas por sus miembros a partir del moldeamiento verbal y el mantenimiento de repertorios conductuales como el modelamiento y la retroalimentación de la familia, las instituciones, las expresiones de arte y comunicación (programas de televisión, series, imágenes, música, entre otras). El punto a demostrar por las autoras tiene que ver con el análisis falso o sesgado de individualizar los problemas de violencia de género e incita analizar las prácticas culturales como la explicación y su relevancia para la instauración de intervenciones y propuestas en distintos ámbitos ajenos al análisis del comportamiento.

\section{CONCLUSIONES}

El objetivo de la investigación consistió en identificar las aportaciones del Análisis de la Conducta acerca de la violencia de género, la primera conclusión tiene que ver con el nulo interés en Latino América. Cabe señalar que otras ciencias han demostrado más interés en la violencia de género que la Psicología. Segunda conclusión, las investigaciones encontradas tratan sobre los principios de la relación triple contingencia en la violencia doméstica y violencia contra la mujer, se explica el control personal por medios de intercambio y la relación de interdependencia personal. Tercera conclusión, la investigación del comportamiento cultural ha explicado la violencia de género con los conceptos metacontigencias, producto agregado y contingencias conductuales entrelazadas (CCE). En relación con la tercera conclusión, la investigación del análisis cultural. Los autores Cihon y Mattaini (2019) mencionan que la Asociación Internacional de Análisis de la Conducta (Association for Behavior Analysis International) conformó un grupo nombrado: ciencia culturo-conductual (culturo-behavior science) con el objetivo de intervenir y estudiar la ciencia de los sistemas culturales y del comportamiento (cultural and behavioral systems science). Cihon y Mattaini (2020) señalan en su libro Behavior Science Perspectives on Culture and Community, Behavior Analysis: Theory, Research and Practice, que la conceptualización de conducta social y cultural de B.F Skinner ha desarrollado el interés por la aplicación de los términos del análisis de la conducta dentro de la Psicología Comunitaria (community psychology), el Análisis de Sistemas Conductuales (behavioral systems analysis), la Gestión del Comportamiento Organizacional (organizational behavior management) y el Análisis Cultural (cultural analysis) bajo la influencia de otras disciplinas como el trabajo social, la educación, la biología, la ciencia de los sistemas, antropología entre otras. No obstante, Baia y Sampaio (2019) afirman que los términos empleados en la ciencia culturo-conductual están en desacuerdo. De ahí que, la actividad de la investigación requiere establecer conceptos centrales, adicionales y redefinición con tal de establecer un consenso conceptual y metodológico de los diseños de investigación. Dicha conclusión coincide con Glenn et al. (2016) los autores realizaron un trabajo en que se discutieron los términos: metacontigencias, linaje conductual, su diferencia con la relación triple continencias, entre otros y los aspectos metodológicos para el estudio de la cultura en entornos ecológicos y su medición (refiere dentro de las instituciones y su actividad en tiempo real) en contra posición de las investigaciones experimentales que se caracterizan por el control de las variables dentro de un ambiente artificial.

Cuarta conclusión, el concepto de género es admisible dentro del Análisis Conductual y es una aportación adicional para las otras ciencias dedicadas a los estudios de género. La propuesta de Ruiz (1995) readquiere interés en la actualidad debido a que el género desde esta perspectiva, conlleva conocer los principios del comportamiento y su influencia contextual, la persona explicada con base a su historia personal y su relación con la cultura. Por otro 
lado, los aportes de la Psicología en general afectan el Feminismo por sus explicaciones individuales en un lenguaje de carácter privado e interno que rechaza y niega la influenza externa de los aspectos políticos. Por lo que, se enfatiza la afectación de ignorar la autoridad, la influencia del sistema político y su estructura. Además, de la creación de términos alusivos a la patología para demeritar los objetivos del Feminismo.

Quinta conclusión, varios autores apuntalan a considerar: a) Identificar, reconocer y aminorar las prácticas sexistas, machistas y misóginas personales y profesionales, b) Realizar investigación en entornos reales y aplicadas para tratar la violencia de género y la violencia contra la mujer, c) Integrar al Feminismo en el Análisis de la Conducta para atender el problema, d) Desarrollar intervenciones y procedimientos en las organizaciones e instituciones gubernamentales para reducir sistemáticamente la violencia de género y su tipología y e) Integración de conceptos provenientes de otros marcos teóricos y conceptuales propios de otros disciplinas (Myers, 1995; Bell y Naugle, 2005; Sasson y Paul, 2014; Baires y Koch, 2020; Cihon y Mattaini, , 2020). Con el proposito de terminar, enfatizamos la relevancia del tema por sus efectos sociales y económicos debido a que la noción común de la violencia de género y la violencia contra la mujer reside en el análisis individual que omite los aspectos culturales (sistema social, su estructura y prácticas). 


\section{REFERENCIAS}

Baia, F., \& Sampaio, A. (2019). Distinguishing Units of Analysis, Procedures, and Processes in Cultural Selection: Notes on Metacontingency Terminology. Behavior and Social Issues, 28, 204-220. doi:https://doi.org/10.1007/s42822019-00017-8

Baires, N. A., \& Koch, S. D. (2020). The Future Is Female (and Behavior Analysis): A Behavioral Account of Sexism nd How Behavior Analysis Is Simultaneously Part of the Problema nd Solution. Behavior Analysis in Practice, 13, 253-262. doi:https://doi.org/10.1007/s40617-019-00394-x

Barrera-Torres, O., Gajardo-Ayala, D., Bautista-Ramírez, C., \& Jiménez-Ríos, R. J. (2021). Racismo y Prejuicio: Una Revisión de la Literatura en Revistas Latinoamericanas desde una Perspectiva Analítico-Conductual. Revista Innovación Digital Y Desarrollo Sostenible, 2(1), 83-93. doi:https://doi.org/10.47185/27113760.v1n2.32

Barriga, N. (2020). Una aproximación teórica a las olas del feminismo: la cuarta ola. Revista Multidisciplinar de Estudios de Género, 5(2), 121-146. doi:https://doi.org/10.20318/femeris.2020.5387

Bell, K. M., \& Naugle, A. E. (2005). Understanding Saty/Leave Decisions in Violent Relationships: A Behavior Analytic Approach. Behavior and Social Issues, 14 , 21-46. doi:https://doi.org/10.5210/ bsi.v14i1.119

Cihon, T., \& Mattaini, M. (2019). Editorial: Emerging Cultural and Behavioral Systems Science. Perspectives on Behavior Science, 42, 699-711. doi:https://doi.org/10.1007/s40614-01900237-8

Cihon, T., \& Mattaini, M. (2020). Behavior Science Perspectives on Culture and Community (First ed.). Springer. doi:https://doi.org/10.1007/ 978-3-030-45421-0
Comisión Economica para América Latina y el Caribe. (2019). Observatorio de Igualdad de Género de América Latina y el Caribe. Obtenido de Muerte de mujeres ocasionada por su pareja o ex-pareja íntima: https://oig.cepal.org/es/indicadores/$\mathrm{m} \mathrm{u}$ e r t e - m u j e res - o c a s i o n a da-su-pareja-o-ex-pareja-intima

Cooper, J., Heron, T., \& Heward, W. (2007). Applied Behavior Analysis (Second ed.). New Jersey, USA: Pearson.

Freixa, B. E. (2003). ¿Qué es conducta? International Journal of Clinical and Health Psychology, 3(3), 595-613.

Gleen, S. S. (1989). Verbal behavior and cultural practices. Behavior Analysis and Social Action, 7(1), 10-15. doi:https://doi.org/10.1007/BF03406102

Glenn, S. S. (1991). Contigencies and metacontigencies: Relations among behavioral, cultural, and biological evolution. En P. A. Lamal, Series in health psychology and behavioral medicine. Behavioral analysis of societies and cultural practices (págs. 39-73). Hemisphere Publishing Corporation.

Glenn, S. S., Malott , M. E., Andery, M. A., Benvenuti, M., Houmanfar, R. A., Sandaker, I., . . . Vasconcel, L. A. (2016). Toward Consistent Terminology in a Behaviorist Approach to Cultural Analysis. Behavior and Social Issues, 11-27. doi:https://doi.org/10.5210/bsi.v25i0.6634

González García, M. A. (2017). Breve recorrido por la historia del Feminismo. HistoriaAgenda(35), 106-113. Obtenido de http://revistas.unam.mx /index.ph p/historiagenda/article/view/65416/57344

Instituto Nacional de Estadística y Geografía. (2016). Encuesta Nacional sobre la Dinámica de las Relaciones en los Hogares (ENDIREH). Obtenido de Encuesta Nacional sobre la Dinámica de las Relaciones en los Hogares (ENDIREH) 2016: https://www.inegi.org.mx/programas/endireh/2016/ 
Instituto Nacional de Estadística y Geografía. (2021). Encuesta Nacional de Seguridad Pública Urbana (ENSU). Obtenido de https://www.inegi.org.mx /programas/ensu/

Luke, M., \& Alavosius, M. (2012). Impacting Community Sustainability through Behavior Change: A Research Framework. Behavior and Social Issues, 54-79. doi:https://doi.org/10.5210/bsi.v21i0.3938

Matsuda, K., García, Y., Catagnus, R., \& Ackerlund, J. (2020). an Behavior Analysis Help Us Understand and Reduce Racism? A review of the Current Literature. Behavior Analysis in Practice, 13, 336-347.doi:https://doi.org/10.1007/s40617020-00411-4

Miller, K. B., Lund, E., \& Weatherly, J. (2012). Applying Operant Learning To The Stay-Leave Decision In Domestic Violence. Behavior and Social Issues, 21, 135-151. doi:https://doi.org/10.5210/bsi.v21i0.4015

Myers, D. L. (1995). Eliminating the battering of women by men: Some considerations for behavior analysis. Journal of Applied Behavior Analysis, 28(4), 493-507. doi:https://doi.org/10.1901/jaba.1995.28-493

ONU Mujeres. (2019). Oficina de las Naciones Unidas contra la Droga y el Delito. ONU Mujeres. Obtenido de https://www.unodc.org/documents/data-and-analysis/gsh/Booklet_5.pdf

ONU Mujeres. (2020). ntensificación de los esfuerzos para eliminar todas las formas de violencia contra las mujeres y las niñas. ONU Mujeres. Obtenido de https://www.unwomen.org/es/digital-library/publications/2020/07/a-75-274sg-report-ending-violence-against-women-and-girls
Organización de las Naciones Unidas. (20 de Mayo de 2021). ONU Mujeres. Obtenido de Preguntas frecuentes: Tipos de violencia contra las mujeres y las niñas: https://www.unwomen.org/es/what-we-do/ending-violence-againstwomen/faqs/types-of-violence

Organización Mundial de la Salud. (2013). Global and regional estimates of violence against women. Organización Mundial de la Salud. Obtenido de https://www.who.int/publications/i/item/9789241564625

Organización Mundial de la Salud. (20 de Junio de 2013). Organización Paramericana de la Salud. Obtenido de Informe de la OMS destaca que la violencia contra la mujer es "un problema de salud global de proporciones epidémicas": https://www3.paho.org/hq/index.php?option=c o $\mathrm{m}$ c o n t e n t \& v i e $w=a r$ ticle\&id=8817:2013-who-report-highlights-viole nce-against-women-global-health-problem-epid emic-proportions\&ltemid=1926\&lang=es\#: :tex $\mathrm{t}=$ Un\%20informe\%20publicado\%20en\%20enero ,entrevistadas\%20repor

Organización Mundial de la Salud. (20 de Mayo de 2021). Organización Mundial de la Salud. Obtenido de Violencia contra la mujer : https://www.who.int/topics/gender_based_violence/es/\#: :text=Las\%20Naciones\% 20Unid a s \% 20 d e fi n e n \% 20 l a, prod u cen\%20en\%20la\%20vida\%20p\%C3\%BAblica

Organización Mundial de la Salud. (9 de Marzo de 2021). Organización Mundial de la Salud. Obtenido de La violencia contra la mujer es omnipresente y devastadora: la sufren una de cada tres mujeres: https://www.who.int/es/news/ite m/09-03-2021-devastatingly-pervasive-1-in-3-women-globally-experience-violen ce

Ruiz, M. R. (1995). B. F. Skinner's Radical Behaviorism: Historical Misconstructions and Grounds for Feminist Reconstructions. Behavior and Social Issues, 5, 29-44. doi:https://doi.org/10.5210/bsi.v5i2.221 
Ruiz, M. R. (2003). Inconspicuous Sources Of Behavioral Control: The Case Of Gendered Practices. The Behavior Analyst, 4(1), 12-16. doi:http://dx.doi.org/10.1037/h0100005

Saini, V., \& Vance, H. (2020). Systemic Racism and Cultural Selection: A Preliminary Analysis of Metacontingencies. Behavior and Social Issues, 29, 52-63. doi:https://doi.org/10.1007/s4282 2-020-00040-0

Sasson, S., \& Paul, L. A. (2014). Labeling Acts of Sexual Violence: What Roles Do Assault Characteristics, Attitudes, and Life Experiences Play? Behavior and Social Issues, 23, 35-49. doi:https://doi.org/10.5210/bsi.v23i0.5215

Segovia. M., Pérez. G. (2021). Femicidios no bajan a pesar de reformas y políticas contra la violencia de género: 131 víctimas entre 2018 y 2020. Centro de investigación Periodística. Obtenido de: https://www.ciperchile.cl/2021/03/07/femic i d i o s - n o - b a j a n - a - p e sar-de-reformas-y-politicas-contra-la-violencia-d e-genero-131-victimas-entre-2018-y-2020/ 